\title{
Pain Assessment in People With Dementia: Remaining Controversies
}

\author{
Mohammad Rababa ${ }^{1}$ \\ ${ }^{1}$ School of Nursing, Jordan University of Science and Technology, Irbid, Jordan \\ Correspondence: Mohammad Rababa, School of Nursing, Jordan University of Science and Technology, Irbid, \\ Jordan, P O Box 3030, Irbid 22110, Jordan. Tel: 962-2-720-1000. E-mail: mjrababa@just.edu.jo
}

Received: February 14, 2018 Accepted: April 3, 2018 Online Published: April 4, 2018

doi:10.5539/gjhs.v10n5p62 URL: https://doi.org/10.5539/gjhs.v10n5p62

\begin{abstract}
Background: Pain in people with dementia is under recognized, underestimated, and under-treated due to the complexity of pain assessment in this population.

Summary: Self-report tools are the gold standard of pain assessment. However, people with dementia are not always able to clearly and meaningfully self-report their pain. Self-report tools capture the affective experience of pain, which is impaired in people with dementia. Observational pain tools are more useful to assess pain especially in people with advanced dementia who are unable to self-report. Observational pain tools capture the physical component of pain experience, which is unchanged in people with dementia. However, nurses often misunderstand the behavioral changes associated with pain in people with dementia. Also, there is a huge inconsistency in presentation of these behavioral changes associated with pain among people with dementia as behaviors can be due to multiple different unmet needs, pain being one.
\end{abstract}

Keywords: Dementia, pain assessment, self-report tools, observational pain tools, elderly people

\section{Introduction}

The gold standard of pain assessment is verbal self-reporting, but meaningful verbal communication is not always possible for older adults with severe dementia (Lukas et al., 2012). According to Horgas and Miller (2008), the manifestations of pain in people with dementia (PWD) can also be assessed by observing changes in behavior. Changes in behaviors include odd facial expression, facial grimacing, negative vocalization, body guarding, and mood swing. However, neither self-reporting nor observation of changes in behavior are consistently interpreted accurately by nurses in PWD. This misunderstanding and misinterpretation of the behavioral changes contribute to ineffective or poor assessment of pain leading to unrelieved pain in PWD (Kovach, 2013).

Pain in PWD often requires multidimensional assessment to ensure prompt and timely administration of analgesics and other nonpharmacological interventions (Park, Castellanos-Brownn, \& Belcher, 2010). Yet nurses often use assessment tools that underestimate pain because of poor understanding of the differences between the physical and affective aspects of the pain experience and the best method to assess pain in PWD (Kovach, Morgan, Noonan, \& Brondino, 2008).

\section{Physical Pain}

Multiple regions in the brain act together to process the state of pain and they are able to modulate the experience of pain (Hugenschmidt \& Sink, 2015). There are specific types of nerve fibers located in the peripheral sensory system responsible for the transmission of pain signals to the spinal cord and somatosensory cortex (Hugenschmidt \& Sink, 2015). According to the gate theory, the physical component of pain experience caused by an external stimulus is moderated at the level of midbrain and in the spinal cord (Mendell, 2014).

It is important to clearly understand and be aware of possible age-related or cognition-related changes of pain perception when examining and assessing the pain experience in older adults and their ability to self-report pain (Lautenbacher, Hofer, \& Kunz, 2018). Research to date is, however, fraught with inconsistent and contradictory findings. Some research studies have revealed that pain may be less frequent and less severe in PWD suffering from some acute medical conditions (Gibson, 2015; Hadjistavropoulos et al., 2015; Pignataro \& Swisher, 2010). Two recent studies (Riley et al., 2014; Stabell, Stubhaug, Flægstad, \& Nielsen, 2013) have revealed that pain threshold increases with age. However, a recent study revealed that whether there is no change or decrease in pain thresholds as age advances (Leegaard, Lomholt, Thastum, \& Herlin, 2013). Furthermore, another recent study 
(McDonagh et al., 2016) revealed that there is age-related loss in the structure and function of the peripheral nerve and central nervous system (CNS), which are involved in processing the physical component of the pain experience, causing reduced pain sensitivity in older adults.

McDonagh et al. (2016) suggested that age-related changes in the processing and interpretation of pain may imped the sensation of pain among older adults, thereby reducing their ability to self-report pain and putting them at greater risk for poor assessment and ineffective management of pain. However, the change in pain processing during later life is more complex (Monroe, Carter, Feldt, Tolley, \& Cowan, 2012), as showed in another study (Horgas, Nichols, Schapson, \& Vietes, 2007) that older adults become less able to endure strong pain sensations. Age-related physical/sensory changes in the CNS also explain reduced pain tolerance in the older adults (Hadjistavropoulos et al., 2014). In combination, these findings showed that older adults might be more vulnerable for the negative health consequences of strong pain. Although the literature may show various findings regarding the decrease or increase of pain sensitivity with advanced age, it remains the common clinical experience of cognitively intact and communicating older adults that many medical and surgical conditions result in considerable pain in this population. Once can assume that although PWD suffer from loss of short-term memory related to recent experiences of pain, they would feel pain in real time for the same conditions in which cognitively intact people express pain.

Despite several research studies conducted about pain in PWD, it is still not clearly known if they have substantial changes in the physical component of pain experience. Some researchers have hypothesized that the physical component of pain experience may be compromised in PWD due to the disease process of dementia and its related neuropathological changes (Achterberg et al., 2013). Also, according to Hadjistavropoulos et al. (2014), in addition to age-related cognitive impairments, dementia may cause an additional burden of cognitive impairment and associated neurodegenerative loss. Therefore, the physical component of pain experience is compromised. Another consideration is that cognitively intact people often guard painful body parts and reduce their mobility, while PWD cannot cognitively process the cause and effect of movement and other behaviors associated with pain, thereby possibly worsening nociceptive pain.

However, some other research studies revealed that the somatosensory cortex is quite preserved in PWD, and hence the physical component of pain experience is more likely uncompromised in PWD (Achterberg et al., 2013). Two early studies of pain thresholds in PWD confirmed this preservation of physical component of pain experience (Kunz, Lautenbacher, LeBlanc, \& Rainville, 2012). The findings of these studies revealed that there is no significant difference in the pain threshold between PWD and cognitively intact older adults. Therefore, there is ample evidence, that although PWD often are unable to self-report pain, they do experience pain from the same sources as cognitively intact people do.

\section{Affective Pain}

While most pain signals are transmitted through the spinal cord to the midbrain, further study has shown that subcentral mechanisms in the thalamus are also activated and then advance to the reticular activating system, an area that processes input from the cortex, cranial nerves, limbic system, hypothalamus, and locus ceruleus. This finding lends credence to the fact of the affective component of pain experience, because hypothalamus, locus ceruleus, and limbic system are associated with fear, anxiety, primitive emotion, and stress (Wilson, Uhelski, \& Fuchs, 2008).

The limbic system is primarily responsible for the processing of the affective component of pain experience. Further pain-modulating processes occur in the hypothalamus, midbrain, and periaqueductal gray. Also, memory plays a significant role in the processing of pain, fear, anxiety, and expectation (Rudich, Lerman, Gurevich, \& Shahar, 2010). Therefore, with impaired memory there is no anticipatory anxiety and fear associated with impending pain, at the same time, there is no protective effect of guarding as those with dementia continue their usual physical movements, unaware that the pain they perceive is due to moving a limb for example.

As shown previously, it is still unclear if PWD experience significant changes in the physical component of pain experience. However, the affective components of pain experience are often significantly compromised in PWD. In other words, PWD experience less affective pain than cognitively intact older adults. Also, they have lower levels of expectation and arousal for pain (Kovach, 2013). For example, as PWD are anticipating the pain of venipuncture, they do not experience an increase in heart rate, however they experience increased heart rate after venipuncture. And perhaps they experience more distress after venipuncture because their cognitive impairment does not allow them to make sense of the pain, to decrease anxiety by knowing that the action causing pain is now ceased. 
This diminished processing of affective component of pain experience in PWD may be explained by recent neuropathologic studies of dementia that have revealed some degrees of atrophy in the amygdala, along with atrophy in the frontal cortex and neurofibrillary pathology. These regions of the brain are the major components of the limbic system and are mainly responsible for the processing of the affective component of pain experience (Kovach, 2013). Other neuropathologic studies have revealed that the disease process of dementia causes the formation of amyloid plaques in the brain tissues involved in the processing of affective pain including the amygdala, thalamic tracts/nuclei, and hypothalamic tracts/nuclei. These amyloid plaques deform these brain tissues, causing impairment. Furthermore, the psychotic symptoms of dementia may cause further impairments in in the affective pain (Wilson et al., 2008).

In addition to the neuropathological effects and psychotic symptoms of dementia, the affective pain may be compromised by the overall memory loss caused by dementia (Rudich et al., 2010). Memory loss would surely change the affective pain in PWD. However, the change in affective pain in PWD caused by memory loss is still not clearly understood. Some studies suggest that memory loss could decrease the affective pain in PWD by reducing the expectation of pain due to the lack of adaptation to painful stimuli (Rudich et al., 2010). Yet cognitive impairment also prevents the person from understanding cause and effect of pain and making adjustments to ameliorate pain as persons with intact cognitive abilities do.

\section{Pain Assessment}

\subsection{Self-Report Tools}

Although PWD have difficulty self-reporting their pain, they are still able to use some words to express pain intensity especially those with mild to moderate dementia (Horgas et al., 2007; Kovach, 2013; Scherder \& Plooij, 2012). Also, PWD may be able to use self-report tools such as the FACES scale or Visual Analog Scale (VAS) (Kovach, 2013; Scherder \& Plooij, 2012), which are simple and easy to use. These scales mainly measure pain intensity, frequency, and location. The VAS represents a continuum of the subjective feeling of pain to be rated with two opposite ends of extremes. For example, the visual analogue scale would be marked with 'no pain' (0) at one end and 'severe pain' (10) at the other. The VAS is one of the most commonly and frequently used tools to assess pain in older adults (Lin, Lin, Shyu, \& Hua, 2011). Some recent research studies have indicated that older adult patients often prefer using the VAS when given a choice (Hadjistavropoulos et al., 2014; Horgas et al., 2007; Zwakhalen, Hamers, \& Berger, 2007). Consistently, several studies have suggested that self-report tools such as the VAS and Faces Pain Scale are still a reasonable and accurate method to assess pain in older adults with mild dementia (Hadjistavropoulos et al., 2014; Horgas et al., 2007; Sheu et al., 2012; Zwakhalen et al., 2007). Also, up to $90 \%$ of older adults with moderate dementia were recently shown to be able to use the VAS accurately. Further, even in severe dementia, there were still older adults capable of using this instrument (Lin et al., 2011).

\subsection{Observational Pain Tools in Dementia}

When older adults with advanced dementia become unable to report their pain, it is recommended that nurses use observation of pain behaviors to assess pain (Scherder \& Plooij, 2012). Observational pain tools are more likely to capture well-established ingrained spontaneous human expressions of physical pain, rather than relying on affective pain experiences or cognitive interpretation of the sensory experience. These expressions include changes in behavior associated with pain such as facial grimacing or crying out in response to pain stimuli, scratching, pushing, odd position or body guarding, rubbing, bracing, and restlessness (Horgas et al., 2007; Zwakhalen et al., 2007). Observational pain tools capture these changes in behaviors to assess pain in PWD. Therefore, the nurses making decisions about pain assessment and management in PWD based on these changes needs to become familiar and know more about using observational pain tools in PWD. In addition to these behavioral changes, it has been revealed that facial expression is the most common and frequently assessed behavior indicator for pain in PWD (Horgas et al., 2007).

In general, changes in facial expression may help in pain assessment by showing changes associated with that pain is being experienced (Horgas et al., 2007). The facial changes that arise during pain have been studied for many years (Fridlund, 2014; Oosterman, Zwakhalen, Sampson, \& Kunz, 2016). Although some variation has been reported, there is substantial agreement across research studies that facial changes are likely to occur more frequently and intensively when people experience pain (Oosterman et al., 2016; 10; Lin et al., 2011; Zwakhalen \& Hamers, 2012). Studies of pain and aging have demonstrated that the facial expression of pain remains consistent, even among older adults with mild dementia. Moreover, older adults with advanced dementia may have an enhanced facial expression of pain because of diminished inhibitory controls (Oosterman et al. 2016).

The main observational tools available to be used by nurses to assess pain in PWD include: Pain Assessment 
Checklist for Seniors with Limited Ability to Communicate-Dutch PACSLAC-D (Zwakhalen \& Hamers, 2012; Zwakhalen et al., 2007); The Doloplus-2 scale (Chen, Lin, \& Watson, 2010,); Mahoney Pain Scale (MPS) (Mahoney \& Peters, 2008); Certified Nursing Assistant Pain Assessment Tool (CAPT) (Cervo et al., 2007); Non-Communicative Patient's Pain Assessment Instrument (NOPPAIN) (Horgas et al., 2007); Pain Assessment Checklist for Seniors with Limited Ability to Communicate (PACSLAC) (Fuchs-Lacelle \& Hadjistavropoulos, 2004); and Pain Assessment in Advanced Dementia (PAINAD) (Warden, Hurley \&, Volicer, 2003). .

Despite the relatively large number of existing observational tools for pain assessment in PWD, some tools have been rated consistently more effective in literature reviews involving psychometric comparisons. Those tools with consistently positive evaluations include the Doloplus-2 (Chen et al., 2010; Hadjistavropoulos et al., 2014); the PACSLAC (Fuchs-Lacelle \& Hadjistavropoulos, 2004; Zwakhalen et al., 2007; Zwakhalen et al., 2012); the Abbey (Van Iersel, Timmerman, \& Mullie, 2006); and the PAINAD (Warden, Hurley, \& Volicer, 2003). Also, the National Nursing Home Pain Collaborative (NNHPC) has considered the NOPPAIN (Horgas et al., 2007) to have strong psychometric properties. However, there is still no one tool that meets all purposes, and clinicians must consider the evidence and clinical usefulness of recommended tools for their specific population and setting. Many studies have examined psychometric properties across scales and investigated unique characteristics of tools that may make them stronger or weaker from a clinical perspective.

\subsection{Psychometric Properties}

\subsubsection{Reliability}

All previously mentioned observational pain tools were tested in nursing homes, and the majority of them had either strong or good internal consistency with an average range $(\alpha=0.75-0.92)$ (Chen et al., 2012; Fuchs-Lacelle \& Hadjistavropoulos, 2004; Horgas et al., 2007; Mahoney \& Peters, 2008; Zwakhalen et al., 2007; Zwakhalen et al., 2012). Two recent studies (Chen et al., 2012; Lin et al., 2011) reported moderate to very high test-retest reliability for the Doloplus-2 and the PAINAD, respectively. The inter-rater reliability of the MOBID, NOPPAIN, PACSLAC, MPS, and PACSLAC-D scales are considered moderate to very high with an average range $(\kappa=0.53$ $0.94)$, indicating that two different nurses rated the behaviors similarly. The inter-rater reliability of the Doloplus-2 scale and PAINAD is high $(\mathrm{ICC}=0.86)$ (Chen et al., 2012; Lin et al., 2011). However, the inter-rater reliability of two tools, the PAINAD and PACLAC, has been tested in nursing homes in both pleasant and unpleasant situations. It is important for the assessment tools to be administered at different times of day and during different situations because pain is not constant; it varies throughout the day and according to level of activity (Horgas \& Miller, 2008).

\subsubsection{Validity}

The validity was not established for all observational pain tools in the original studies. Since some observational tools were developed without direct input from PWD and they were used alone, it is difficult to establish their validity. However, some researchers have conducted validity tests for their observational tools, such as the PACSLAC and NOPPAIN (Fuchs-Lacelle \& Hadjistavropoulos, 2004; Horgas et al., 2007). The validity of the PACSLAC was assessed by calculating the correlation between the level of pain intensity measured by the VAS and the total PACSLAC scores $(\mathrm{r}=0.54, \mathrm{p}=.001)$ (Fuchs-Lacelle \& Hadjistavropoulos, 2004). Validity of the NOPAIN was assessed by calculating its correlation with a Numeric Rating Scale (NRS) $(r=0.66, p \leq 0.05)$ and a Verbal Descriptor Scale (VDS) ( $\mathrm{r}=0.66, \mathrm{p} \leq 0.05)$ (Horgas et al., 2007).

No study has tested the sensitivity and specificity tests for the NOPPAIN, PACSLAC, and Abbey on nursing home residents. A recent study (Jordan, Regnard, O'Brien, \& Hughes, 2012) stated clearly that although the PAINAD tool has a high sensitivity ( $92 \%)$, it has a low specificity (62\%) for pain. The low specificity is due to the fact that the PAINAD tool also measures distress not caused by pain. Although Chen et al. address the sensitivity and specificity of the Doloplus-2 scale, they do not report numerical values of both tests. According to Chen et al. the Doloplus-2 scale had high sensitivity and low specificity in pain assessment. Table 1 below summarize and compare between the psychometric properities of the Observational pain tools. 
Table 1. Observational tools used to Assess Pain

\begin{tabular}{|c|c|c|c|c|c|c|c|}
\hline Instrument & The study & $\begin{array}{l}\text { Inter-rater } \\
\text { reliability }\end{array}$ & $\begin{array}{l}\text { Test-retest } \\
\text { reliability }\end{array}$ & $\begin{array}{l}\text { Internal } \\
\text { consistency }\end{array}$ & Validity & Specificity & Sensitivity \\
\hline PAINAD & Warden et al., 2003 & $\mathrm{ICC}^{\mathrm{a}}=0.83$ & $\mathrm{ICC}=0.71$ & & & & \\
\hline CPAT & Cervo et al., 2007 & $\mathrm{ICC}=0.57$ & $\mathrm{ICC}=0.50$ & & $r=0.25$ & & \\
\hline NOPPAIN & Horgas et al., 2007 & $\kappa^{\mathrm{b}}=0.87$ & & $\alpha^{\mathrm{c}}=.94$ & $r=0.66$ & & \\
\hline MOBID & Husebo et al., 2009 & $\kappa=0.53-.94$ & & & & & \\
\hline PACSLAC & $\begin{array}{l}\text { Fuchs-Lacelle \& } \\
\text { Hadjistavropouls, } 2004\end{array}$ & $\begin{array}{l}\kappa=0.78 \\
0.95\end{array}$ & & $\alpha=0.92$ & $r^{e}=.54$ & & \\
\hline PACSLAC-D & $\begin{array}{l}\text { Zwakhalen et al., } 2012 \\
\text { \& Zwakhalen et al., } \\
2007\end{array}$ & $\begin{array}{l}\kappa=0.77 \\
0.96\end{array}$ & & $\alpha=0.82-0.86$ & & & \\
\hline $\begin{array}{l}\text { Doloplus-2 } \\
\text { scale }\end{array}$ & $\begin{array}{l}\text { Hadjistavropoulos et } \\
\text { al., } 2012 \& \text { Chen et al., } \\
2010\end{array}$ & $\mathrm{ICC}=0.86$ & $\mathrm{ICC}=0.89$ & $\alpha=0.75$ & & & \\
\hline MPS & $\begin{array}{l}\text { Mahoney \& Peters, } \\
2008\end{array}$ & $\begin{array}{l}\kappa= \\
0.71-0.74\end{array}$ & & $\alpha=0.76$ & $\kappa=.86$ & $86 \%$ & $98 \%$ \\
\hline
\end{tabular}

$\mathrm{ICC}^{\mathrm{a}}=$ intra-class coefficient;

$\alpha^{\mathrm{c}}=$ Cronbach's alpha; acceptable if $\alpha=0.70$, high $\alpha \geq 0.80$;

$\kappa^{\mathrm{b}}=$ Kappa statistics; $\leq 0$ poor, .01-.20=light, .21-.40=fair, .41-.60=moderate, $.61-.80=$ high, and $.81-1=$ very high;

$r^{e}=$ Correlation; validity is good at $p \leq 0.05$.

\section{Remaining Controversy}

The best assessment method of Pain in PWD is still controversial. After reviewing the literature, it has been revealed that there are three different positions on what the best assessment method has to be used for pain assessment in PWD. It is not clear if self-report tools are sufficient to assess pain in PWD if they can still self-report. For people with severe dementia or communication deficit, observational tools are the best method or strategy to assess pain (Cervo et al., 2010: Mahoney \& Peters, 2008). However, there is inconsistency of behavioral changes associated with pain in PWD. Also, no study identifies specific behavioral changes that certainly indicate the presence of pain in PWD. Nurses often use either a self-report tool or an observational tool to assess pain in PWD. However, some studies have revealed that nurses use a combination of both tools to assess pain and compare the scores of observational tools with the scores of self-report tools to validate them (Kovach, 2013).

Some studies revealed that self-report tools are the most reliable measure of pain in PWD as long as they are able to self-report pain. For those PWD, it is recommended that self-report of pain attempted regardless of their cognitive impairment (Kovach, 2013), keeping in mind that it may not be enough alone. Nurses should be responsible for fostering productive discussions about pain with PWD. For example, nurses should be trained in how to determine the patient's preferred terminology of pain. PWD need to be frequently asked about some pain related term such as discomfort, aching, or soreness. Hence, PWD no longer deny their pain after nurses reword the question with other pain related terms. For example, nurses could ask, "Do you hurt anywhere?" or "Are you uncomfortable?" to rule out pain or discomfort. Once a nurse establishes the preferred pain terminology for PWD he or she needs to document it, communicate it to other healthcare providers, and use it throughout the treatment trajectory (Snow et al., 2004). Another factor that could help foster pain self-reporting is to allow sufficient time for PWD to process the question asked and then formulate a response (Herr \& Garand, 2001). Findings in a recent study (Snow et al.) indicated that $83 \%$ of PWD in a nursing home were able to respond to a self-report tool. According to the study, self-report tools alone are more sufficient to assess pain in communicating PWD. In this particular case, using additional types of pain assessment may cause confusion and inconsistencies among nurses. Also, self-report pain tools are easier to use by nurses, more understandable by patients, and practical and known across the clinical settings (Gregory \& Richardson, 2014). It is worth trying to use self-report tools, because some PWD will be able to use them, it promotes dignity by giving them the chance to use it. 
In contrary, some studies have reported that self-report alone is insufficient, and observational pain assessment tools are needed for PWD even if they are communicating. Impaired memory and language disabilities caused by dementia impede the ability of PWD to meaningfully self-report their painful experiences. Other confounding factors include delirium, altered level of consciousness, presence of an endotracheal tube, sedatives, and neuromuscular blocking agents (Herr, Coyne, McCaffery, Manworren, \& Merkel, 2011). Furthermore, the pathologic changes in dementia seriously affect the ability of those with advanced stages of disease to communicate pain effectively and meaningfully. Damage to the central nervous system affects memory, language, and higher-order cognitive processing necessary to communicate the experience (Herr et al., 2011).

There is also a different third position on the best assessment method of pain in PWD adopted by American Society for Pain Management (ASPM). According to ASPM, there is no single pain assessment method sufficient by itself. Therefore, both self-report and observation pain tools are recommended by the ASPM for PWD, regardless of their ability to self-report. Also, according to a research study (Gilmore-Bykovskyi \& Bowers, 2013), pain management is a multidimensional clinical process requiring thorough and systematic assessment, using a combination of both self-report and observational pain tools. Furthermore, According to Monroe, Gore, Chen, Mion, and Cowan (2012), the multidimensional model of pain assessment suggests incorporating neuroimaging techniques into the assessment process of pain. This leads to expand nurses' understanding of pain perception in people with dementia as well as the structural and functional changes, which happen in their brain. Also, the multidimensional model of pain assessment succeeds in interpreting the complex relationship among dementia pathology, brain activation, and pain reports.

\section{Conclusion}

Pain in PWD is still unrelieved due to the complexity of pain assessment, cognitive impairment, and communication deficit in PWD. To date, there is no single tool captures both components of pain experience in PWD. Furthermore, affective component of pain experience measured by self-report tools is compromised in PWD. Moreover, there is inconsistency of behavioral changes among PWD thereby making the assessment of the physical component of pain experience complicated. Therefore, it is still controversial whether self-report tools, observational pain tools, or self-report tools and observational pain tools combined together is sufficient for pain assessment in PWD. Future research is recommended to properly address both components of pain experience (physical and affective) in PWD together in one assessment model to obtain more comprehensive pain-assessment protocol. Also, a new paradigm for pain assessment is needed which includes neuroimaging techniques.

\section{Competing Interests Statement}

The author declares that there are no competing or potential conflicts of interest.

\section{References}

Achterberg, W. P., Pieper, M. J., van Dalen-Kok, A. H., De Waal, M. W., Husebo, B. S., Lautenbacher, S., ... \& Corbett, A. (2013). Pain management in patients with dementia. Clinical Interventions In Aging, 8, 1471-1482. https://doi.org/10.2147/CIA.S36739

Cervo, F. A., Raggi, R. P., Bright-Long, L. E., Wright, W. K., Rows, G., Torres, A. E., ... \& Komaroff, E. (2007). Use of the certified nursing assistant pain assessment tool (CPAT) in nursing home residents with dementia. American Journal of Alzheimer's Disease \& Other Dementias ${ }^{\circledR}, \quad 22(2), \quad 112-119$. https://doi.org/10.1177/1533317506298907

Chen, Y. H., Lin, L. C., \& Watson, R. (2010). Validating nurses' and nursing assistants' report of assessing pain in older people with dementia. Journal of Clinical Nursing, 19(1-2), 42-52. https://doi.org/ 10.1111/j.1365-2702.2009.02950.x.

Fridlund, A. J. (2014). Human facial expression: An evolutionary view. San Diego: Academic Press.

Fuchs-Lacelle, S., \& Hadjistavropoulos, T. (2004). Development and preliminary validation of the pain assessment checklist for seniors with limited ability to communicate (PACSLAC). Pain Management Nursing, 5(1), 37-49. https://doi.org/10.1016/j.pmn.2003.10.001

Gibson, S. J. (2015). The Pain, Emotion and Cognition Nexus in Older Persons and in Dementia. In Pain, Emotion and Cognition (pp. 231-247). Springer, Cham. https://doi.org/10.1007/978-3-319-12033-1_15

Gilmore-Bykovskyi, A. L., \& Bowers, B. J. (2013). Understanding nurses' decisions to treat pain in nursing home residents with dementia. Research In Gerontological Nursing, 6(2), 127-138. https://doi.org/10.3928/19404921-20130110-02 
Gregory, J., \& Richardson, C. (2014). The use of pain assessment tools in clinical practice: a pilot survey. Journal of Pain relief, 3(2), 140-146. https://doi.org/ 10.4172/2167-0846.100140

Hadjistavropoulos, T., Herr, K., Prkachin, K. M., Craig, K. D., Gibson, S. J., Lukas, A., \& Smith, J. H. (2014). Pain assessment in elderly adults with dementia. The Lancet Neurology, 13(12), 1216-1227. https://doi.org/10.1016/S1474-4422(14)70103-6

Herr, K. A., \& Garand, L. (2001). Assessment and measurement of pain in older adults. Clinics in Geriatric Mmedicine, 17(3), 457-478. https://doi.org/10.1016/S0749-0690(05)70080-X

Herr, K., Coyne, P. J., McCaffery, M., Manworren, R., \& Merkel, S. (2011). Pain assessment in the patient unable to self-report: position statement with clinical practice recommendations. Pain Management Nursing, 12(4), 230-250. https://doi.org/10.1016/j.pmn.2011.10.002

Horgas, A., \& Miller, L. (2008). Pain assessment in people with dementia. AJN The American Journal of Nursing, 108(7), 62-70. https://doi.org/10.1097/01.NAJ.0000325648.01797.fc

Horgas, A. L., Nichols, A. L., Schapson, C. A., \& Vietes, K. (2007). Assessing pain in persons with dementia: relationships among the non-communicative patient's pain assessment instrument, self-report, and behavioral observations. Pain Management Nursing, 8(2), 77-85. https://doi.org/10.1016/j.pmn.2007.03.003

Hugenschmidt, C. E., \& Sink, K. M. (2015). No pain, functional gain: the importance of pain management in older adults with cognitive impairment. Pain, 156(8), 1377. https://doi.org/10.1097/j.pain.0000000000000232

Jordan, A., Regnard, C., O’Brien, J. T., \& Hughes, J. C. (2012). Pain and distress in advanced dementia: choosing the right tools for the job. Palliative Mmedicine, 26(7), 873-878. https://doi.org/10.1177/0269216311412227

Kunz, M., Lautenbacher, S., LeBlanc, N., \& Rainville, P. (2012). Are both the sensory and the affective dimensions of pain encoded in the face? Pain, 153(2), 350-358. https://doi.org/10.1016/j.pain.2011.10.027

Kovach, C. R. (2013). Assessing pain and unmet need in patients with advanced dementia: the role of the Serial Trial Intervention (STI). In Handbook Of Pain And Palliative Care (pp. 131-144). Springer, New York, NY. https://doi.org/10.1007/978-1-4419-1651-8_10

Kovach, C. R., Morgan, S., Noonan, P. E., \& Brondino, M. (2008). Using principles of diffusion of innovation to improve nursing home care. Journal of Nursing Care Quality, 23(2), 132-139. https://doi.org/10.1097/01.NCQ.0000313762.79396.ec

Lautenbacher, S., Hofer, W., \& Kunz, M. (2015). Age Differences in Decoding Pain from the Facial Expression of Healthy Individuals and Patients with Dementia. Pain Medicine, 17(4), 685-691. https://doi.org/ $10.1111 /$ pme. 12927

Leegaard, A., Lomholt, J. J., Thastum, M., \& Herlin, T. (2013). Decreased pain threshold in juvenile idiopathic arthritis: a cross-sectional study. The Journal of Rheumatology, 40(7), 1212-1217. https://doi.org/10.3899/jrheum.120793

Lin, P. C., Lin, L. C., Shyu, Y. I. L., \& Hua, M. S. (2011). Predictors of pain in nursing home residents with dementia: a cross - sectional study. Journal of Clinical Nursing, $20(13$ - 14), 1849-1857. https://doi.org/10.1111/j.1365-2702.2010.03695.x

Lukas, A., Schuler, M., Fischer, T. W., Gibson, S. J., Savvas, S. M., Nikolaus, T., \& Denkinger, M. (2012). Pain and dementia. Zeitschrift für Gerontologie und Geriatrie, 45(1), $45-49$. https://doi.org/10.1007/s00391-011-0272-4

Mahoney, A. E., \& Peters, L. (2008). The Mahoney Pain Scale: examining pain and agitation in advanced dementia. American Journal of Alzheimer's Disease \& Other Dementias ${ }^{\circledR}, \quad 23(3), \quad 250-261$. https://doi.org/10.1177/1533317508317524

McDonagh, B., Scullion, S. M., Vasilaki, A., Pollock, N., McArdle, A., \& Jackson, M. J. (2016). Ageing-induced changes in the redox status of peripheral motor nerves imply an effect on redox signalling rather than oxidative damage. Free Radical Biology and Medicine, 94, 27-35. https://doi.org/10.1016/j.freeradbiomed.2016.02.

Mendell, L. M. (2014). Constructing and deconstructing the gate theory of pain. PAIN®, 155(2), $210-216$. https://doi.org/10.1016/j.pain.2013.12.010

Monroe, T., Carter, M., Feldt, K., Tolley, B., \& Cowan, R. L. (2012). Assessing advanced cancer pain in older adults with dementia at the end - of - life. Journal of Advanced Nursing, 68(9), 2070-2078. 
https://doi.org/10.1111/j.1365-2648.2011.05929.x

Oosterman, J. M., Zwakhalen, S., Sampson, E. L., \& Kunz, M. (2016). The use of facial expressions for pain assessment purposes in dementia: a narrative review. Neurodegenerative Ddisease Management, 6(2), 119-131. https://doi.org/10.2217/nmt-2015-0006

Park, J., Castellanos-Brown, K., \& Belcher, J. (2010). A review of observational pain scales in nonverbal elderly with cognitive impairments. Research on Social Work Practice, 20(6), 651-664. https://doi.org/10.1177/1049731508329394

Pignataro, R. M., \& Swisher, A. K. (2010). Chemotherapy induced peripheral neuropathy: risk factors, pathophysiology, assessment, and potential physical therapy interventions. Rehabilitation Oncology, 28(2), 10-18. https://doi.org/ 10.3389/fnmol.2017.00174

Riley, J. L., Cruz-Almeida, Y., Glover, T. L., King, C. D., Goodin, B. R., Sibille, K. T., ... \& Redden, D. T. (2014). Age and race effects on pain sensitivity and modulation among middle-aged and older adults. The Journal of Pain, 15(3), 272-282. https://doi.org/10.1016/j.jpain.2013.10.015

Rudich, Z., Lerman, S. F., Gurevich, B., \& Shahar, G. (2010). Pain specialists' evaluation of patient's prognosis during the first visit predicts subsequent depression and the affective dimension of pain. Pain Medicine, 11(3), 446-452. https://doi.org/10.1111/j.1526-4637.2009.00795.x

Scherder, E. J., \& Plooij, B. (2012). Assessment and management of pain, with particular emphasis on central neuropathic pain, in moderate to severe dementia. Drugs \& Aging, 29(9), 701-706. https://doi.org/10.1007/s40266-012-0001-8

Sheu, E., Versloot, J., Nader, R., Kerr, D., \& Craig, K. D. (2011). Pain in the elderly: validity of facial expression components of observational measures. The Clinical Journal of Pain, 27(7), 593-601. https://doi.org/10.1097/AJP.0b013e31820f52e1

Snow, A. L., Weber, J. B., O’Malley, K. J., Cody, M., Beck, C., Bruera, E., ... \& Kunik, M. E. (2004). NOPPAIN: a nursing assistant-administered pain assessment instrument for use in dementia. Dementia and Geriatric Cognitive Disorders, 17(3), 240-246. https://doi.org/10.1159/000076446

Stabell, N., Stubhaug, A., Flægstad, T., \& Nielsen, C. S. (2013). Increased pain sensitivity among adults reporting irritable bowel syndrome symptoms in a large population-based study. PAIN®, 154(3), 385-392. https://doi.org/10.1016/j.pain.2012.11.012

van Iersel, T., Timmerman, D., \& Mullie, A. (2006). Introduction of a pain scale for palliative care patients with cognitive impairment. International Journal of Palliative Nursing, 12(2), 54-59. https://doi.org/10.12968/ijpn.2006.12.2.20531

Warden, V., Hurley, A. C., \& Volicer, L. (2003). Development and psychometric evaluation of the Pain Assessment in Advanced Dementia (PAINAD) scale. Journal of the American Medical Directors Association, 4(1), 9-15. https://doi.org/10.1097/01.JAM.0000043422.31640.F7

Wilson, H. D., Uhelski, M. L., \& Fuchs, P. N. (2008). Examining the role of the medial thalamus in modulating the affective dimension of pain. Brain Research, 1229, 90-99. https://doi.org/10.1016/j.brainres.2008.06.009

Zwakhalen, S. M., Hamers, J. P., \& Berger, M. P. (2007). Improving the clinical usefulness of a behavioural pain scale for older people with dementia. Journal of Advanced Nursing, 58(5), 493-502. https://doi.org/10.1111/j.1365-2648.2007.04255.x

Zwakhalen, S. M., Hof, C. E., \& Hamers, J. P. (2012). Systematic pain assessment using an observational scale in nursing home residents with dementia: exploring feasibility and applied interventions. Journal of Clinical Nursing, 21(21-22), 3009-3017. https://doi.org/10.1111/j.1365-2702.2012.04313.x

\section{Copyrights}

Copyright for this article is retained by the author(s), with first publication rights granted to the journal.

This is an open-access article distributed under the terms and conditions of the Creative Commons Attribution license (http://creativecommons.org/licenses/by/4.0/). 